\title{
Adult Respiratory Distress Syndrome Induced by a Chinese Medicine, Kamisyoyo-San
}

\author{
Yutaro Shiota, James G. Wilson***, Hitomi Matsumoto, Mitsugu Munemasa*, Midori Okamura*, \\ Junichiro Hiyama*, Masaomi Marukawa*, Tetsuya Ono*, Kiyomi Taniyama** and Hiroto Mashiba* \\ We report the first case of Kamisyoyo-san-induced adult respiratory distress syndrome \\ (ARDS). A 59-year-old female was given Kamisyoyo-san for treatment of seborrheic dermatitis. \\ She then presented with a respiratory illness having clinical, radiologic and functional character- \\ istics of ARDS. Bronchoalveolar lavage fluid showed an increased number of lymphocytes, \\ neutrophils and eosinophils. The lymphocyte stimulation test with Kamisyoyo-san was positive. \\ (Internal Medicine 35: 494-496, 1996)
}

Key words: lymphocyte stimulation test, bronchoalveolar lavage

\section{Introduction}

In Japan, Chinese medicines have been used in various diseases for centuries. More recently, the number of reports of Chinese medicine-induced pneumonitis has gradually increased. We report here a case of adult respiratory distress syndrome (ARDS), which was induced by the Chinese medicine, Kamisyoyo-san. The patient required mechanical ventilation because of severe hypoxemia. To our knowledge, this is the first report of Chinese medicine-induced ARDS.

\section{Case Report}

A 59-year-old female had been well until 6 days before admission, when she started to take hydroxyzine pamoate, minocyclin and a Chinese medicine, Kamisyoyo-san, for treatment of seborrheic dermatitis. One day before admission, she developed high fever, dry cough and dyspnea. Physical examination at the time of hospital admission revealed no abnormalities except fine crackles at the left lung base. Her body temperature was $39.5^{\circ} \mathrm{C}$. Laboratory data revealed a white blood cell count of 5,470 with $73.5 \%$ neutrophils and $10.3 \%$ eosinophils, an erythrocyte sedimentation rate of $55 \mathrm{~mm} / \mathrm{h}$, C-reactive protein (CRP) of $16.3 \mathrm{mg} / \mathrm{dl}$, fibrinogen degradation product (FDP) of $12 \mu \mathrm{g} / \mathrm{dl}$, and hypoxemia $\left(\mathrm{PaO}_{2}: 68.1 \mathrm{mmHg}\right.$; and $\mathrm{PaCO}_{2}: 32.0 \mathrm{mmHg}$ ).

For editorial comment, see p 433.
Tests of anti-Legionella, anti-Mycoplasma and anti-Chlamydia psittaci antibodies were all negative. Sputum culture yielded normal respiratory flora. The findings from routine blood chemistries and urinalysis were normal. Chest X-ray film on admission revealed bilateral diffuse reticular infiltrates. A computed tomographic (CT) scan of the thorax showed bilateral patchy air-space consolidation (Fig. 1). Bilateral small pleural effusions were visible. Cefazolin and rifampicin were administered. On the second hospital day an X-ray film of the chest showed marked progression of the air-space disease (Fig. 2). $\mathrm{PaO}_{2}$ was $48.4 \mathrm{mmHg}$ and $\mathrm{PaCO}_{2}$ was $34.9 \mathrm{mmHg}$ on room air. The patient was admitted to an intensive care unit. An endotracheal. tube was inserted and ventilatory assistance was begun. While the patient was ventilated with $80 \%$ oxygen, with intermittent mandatory ventilation (IMV) of 20 and with positive endexpiratory pressure (PEEP) of $3 \mathrm{~cm}, \mathrm{PaO}_{2}$ was $85.5 \mathrm{mmHg}$ and $\mathrm{PaCO}_{2}$ was $53.1 \mathrm{mmHg}$. On the third hospital day methylprednisolone, $500 \mathrm{mg}$ daily, was started. After 5 days of administration, methylprednisolone was discontinued and prednisolone $(60 \mathrm{mg})$ was begun. On the sixth hospital day a chest X-ray film showed moderate resolution of infiltrates. Bronchoalveolar lavage (BAL) was performed and the recovery of BAL was 53\%; differential count of the white blood cells showed that $18.4 \%$ were macrophages, $35.8 \%$ lymphocytes, $8.6 \%$ eosinophils, and $37.0 \%$ neutrophils. The analysis of surface markers of lymphocytes in the BAL fluid showed that $68.5 \%$ were CD4 positive cells and $6.7 \%$ were CD8 positive cells. The concentration of carcinoembryonic antigen of BAL fluid was markedly elevated $(21.7 \mathrm{ng} / \mathrm{ml})$. Clearing of the pulmonary

\footnotetext{
From the Departments of Clinical Research, *Internal Medicine, **Clinical Pathology, Kure Kyosai Hospital, Kure and ***the Department of Veterans Affairs Medical Center and University of Mississippi Medical Center, Jackson, Miss 


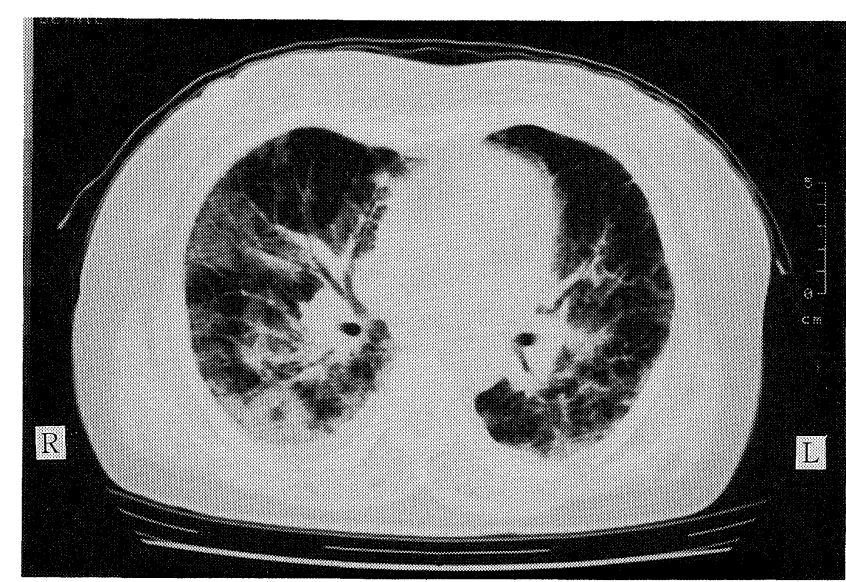

Figure 1. Computed tomography of the chest shows multiple patchy air-space consolidation with a small amount of pleural effusion.

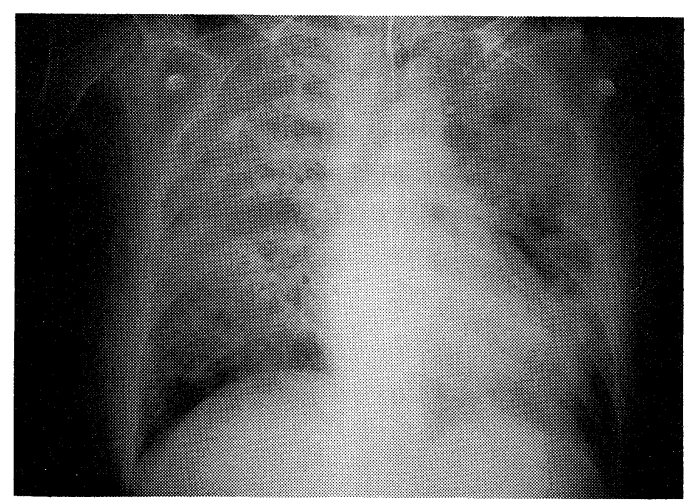

Figure 2. Chest X-ray film on the second hospital day shows marked bilateral air-space consolidation.

infiltrates occurred by the 8th hospital day, and bilateral small infiltrates remained peripherally. The ventilator was discontinued on the 9th hospital day. Lymphocyte stimulation tests with Kamisyoyo-san and other drugs were performed just before the start of methylprednisolone. Only Kamisyoyo-san significantly induced lymphocyte blastogenesis with a stimulation index of 5.2. The diagnosis of Kamisyoyo-san-induced ARDS was then considered to be likely. Prednisolone was tapered and finally discontinued. All of the levels of ESR, CRP, $\mathrm{PaO}_{2}$ and $\mathrm{PaCO}_{2}$ became normal and X-ray film and CT of the thorax showed complete clearing of alveolar opacities at the time of discharge from hospital (Fig. 3).

\section{Discussion}

Chinese medicines generally consist of several different ingredients; some ingredients are commonly shared by different Chinese medicines. Kamisyoyo-san contains 10 ingredients. Because of their mild and gradual therapeutic effects, little

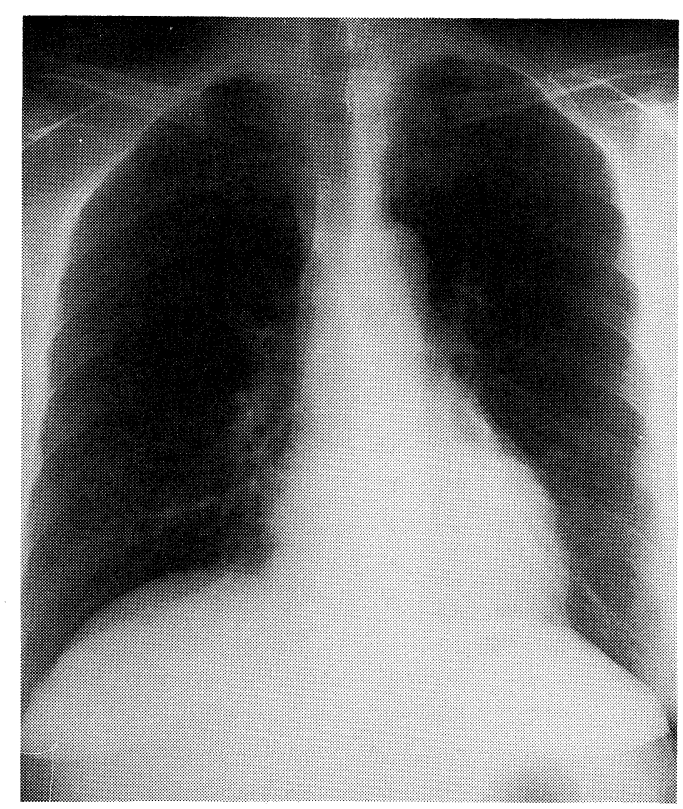

Figure 3. Chest X-ray film at discharge shows complete clearing of alveolar opacities.

attention has been paid to their side effects. Chinese medicineinduced interstitial pneumonitis was reported for the first time in 1989 by Tsukiyama et al (1). To our knowledge, 17 cases of Chinese medicine-induced pneumonitis have been reported up to 1995 (Table 1) (1-7). Provocation tests with the Chinese medicines have been performed in 9 of these cases. Eleven of the cases were induced by Sho-saiko-to and two cases by Saiboku-to. Pneumonia-inducing ingredients of Chinese medicines have been identified in only three cases. Those ingredients include scutellaria root (case 1), scutellaria root, pinellia tuber and bupleum root (case 12) and pinellia tuber (case 16). Kamisyoyo-san consists of Tang-Kuei, peony, gardenia, atractylodes lancea rhizome, hoelen, mentha, bupleurum root, moutan, ginger rhizome and perilla herb and does not contain either scutellaria root or pinellia tuber. In these 17 cases the length of treatment with Chinese medicines prior to the development of symptoms varied from 2 hours to 1 year, with a mean duration 4.6 months. Signs and symptoms developing in these patients included fever, cough and dyspnea on exercise. BAL lymphocytosis was found in all 8 cases studied. In 7 of the 8 cases, the ratio of CD4 to CD8 lymphocytes of BAL fluid was less than 1.0. The present report, however, indicates that lung injury in this case was associated with a significant increase in the percentage of CD4-positive rather than CD8-positive lymphocytes. The reasons that the CD4/CD8 ratios of lymphocytes in BAL fluid are increased in some cases and not in others are unknown. In this case the corticosteroid therapy might have increased the CD4/CD8 ratio of lymphocytes in the BAL fluid.

In the context of marked respiratory distress, diffuse pulmonary infiltration on chest roentgenogram and impairment of 


\section{SHIOTA et al}

Table 1. Age, Sex, Chief Complaints, BAL Findings, Diagnostic Procedures and Treatment of Chinese Medicine-Induced Lung Injury

\begin{tabular}{|c|c|c|c|c|c|c|c|c|c|c|c|}
\hline 2 & Sujiura & 1990 & 61 & M & Sho-saiko-to & Dyspnea on exercise & 1 year & 71 & 0.11 & - & + \\
\hline 3 & Iinuma & 1990 & 46 & M & Sho-saiko-to & Fever & 5 weeks & 66 & 0.04 & - & + \\
\hline 4 & Koyanagi & 1991 & 50 & M & Sho-saiko-to & Dyspnea & 2 weeks & N.D. & N.D. & - & + \\
\hline 7 & Miyazawa & 1991 & 65 & M & Sho-saiko-to & Fever & 3 weeks & 48 & 0.25 & + & N.D. \\
\hline 8 & Kubota & 1991 & 58 & M & Sho-saiko-to & Dyspnea on exercise & 3 months & N.D. & N.D. & + & N.D. \\
\hline 9 & Kubota & 1991 & 50 & $F$ & Juzentaiho-to & Fever, Cough & 6 months & N.D. & N.D. & + & N.D. \\
\hline 10 & Kubota & 1991 & 48 & M I & Keishibukuryou-gan & Dyspnea on exercise & 4 weeks & N.D. & N.D. & + & N.D. \\
\hline 11 & Souda & 1991 & 56 & $\mathrm{~F}$ & Saiboku-to & Fever, Cough & 5 months & N.D. & N.D. & + & N.D. \\
\hline 16 & Maruyama & 1994 & 79 & $\mathrm{~F}$ & Rikkunshi-to & Dyspnea on exercise & 6 months & 45.9 & 0.18 & + & N.D. \\
\hline 17 & Temaru & 1994 & 60 & $\mathrm{~F}$ & Saiboku-to & Dyspnea & 2 hours & N.D. & N.D. & + & + \\
\hline 18 & present case & & 59 & $\mathrm{~F}$ & Kamisyoyo-san & Dyspnea, Fever & 4 days & 35.8 & 10.2 & + & N.D. \\
\hline
\end{tabular}

LST: lymphocyte stimulation test, N.D.: not determined, D.M.: discontinuation of medicine.

oxygen transport, this patient's disorder is in accordance with the adult respiratory distress syndrome (8). The increased number of eosinophils in the peripheral blood and bronchoalveolar lavage fluid suggests that eosinophilic pneumonia might have been induced by Kamisyoyo-san initially, followed by the development of ARDS. Due to the lack of histological documentation of the lesion at early stages of the disease, we can not confirm this.

There are several previous reports of drug-induced ARDS $(9,10)$. This is the first report of ARDS induced by Chinese medicines. Hereafter appropriate consideration must be given to the possible occurrence of medication-induced lung injury in patients being treated with Chinese medicines.

\section{References}

1) Tsukiyama K, Tasaka $Y$, Nakajima $M$, et al. A case of pneumonitis due to Sho-Saiko-To. Nippon Kyobu Shikkan Gakkai Zasshi (Jpn J Thorac Dis) 27: 1556, 1989 (Abstract in English).

2) Temaru R, Yamashita N, Matsui S, Ohta T, Kawasaki A, Kobayashi M. A case of drug induced pneumonitis caused by Saiboku-To. Nippon
Kyobu Shikkan Gakkai Zasshi (Jpn J Thorac) 32: 485, 1994 (Abstract in English).

3) Imokawa $\mathrm{S}$, Sato $\mathrm{A}$, Taniguchi $\mathrm{M}$, et al. A case of Sho-Saiko-to induced pneumonitis and the review of literature. Nippon Kyobu Rinshou (Jpn J Chest Dis) 1: 53, 1992 (Abstract in English).

4) Kubota M, Hanada N, Masuda C, et al. Three cases of pneumonitis caused by herb drugs. Kokyu (Resp Res) 10: 475, 1991 (Abstract in English).

5) Tomioka H. Kanpo-Hozai induced pneumonitis. Saishin Igaku 47: 84, 1992 (Abstract in English).

6) Daibo A, Yoshida Y, Kitazawa S, Kosaka Y, Bando T, Sudo M. A case of pneumonitis and hepatic injury caused by a herbal drug (sho-saiko-to). Nippon Kyobu Shikkan Gakkai Zasshi (Jpn J Thorac Dis) 30: 1583, 1992 (Abstract in English).

7) Maruyama Y, Maruyama M, Takada T, Haraguchi M, Uno K. A case of pneumonitis due to Rikkunshi-to. Nippon Kyobu Shikkan Gakkai Zasshi (Jpn J Thorac Dis) 32: 1, 1994 (Abstract in English).

8) Murray JF, Matthay MA, Luce JM, Flick MR. An expanded definition of the adult respiratory distress syndrome. Am Rev Respir Dis 138: 720, 1988.

9) Howard JJ, Mohsenifer Z, Simons SM. Adult respiratory distress syndrome following administration of lidocaine. Chest 81: 644, 1982.

10) Poe RH, Condemi JJ, Weinstein SS, Schuster RJ. Adult respiratory distress syndrome related to ampicillin sensitivity. Chest 77: 449, 1980. 